\title{
Anaplastic lymphoma kinase (ALK 1) staining and molecular analysis in inflammatory myofibroblastic tumours of the bladder: a preliminary clinicopathological study of nine cases and review of the literature
}

\author{
Alex Freeman ${ }^{1}$, Nicola Geddes ${ }^{2}$, Philippa Munson ${ }^{1}$, Jean Joseph ${ }^{1}$, Pramila Ramani ${ }^{2}$, \\ Ann Sandison ${ }^{3}$, Cyril Fisher ${ }^{4}$ and M Connie Parkinson ${ }^{1}$ \\ ${ }^{1}$ Department of Histopathology, University College Hospital, London; ${ }^{2}$ Department of Histopathology, \\ Birmingham Children's Hospital; ${ }^{3}$ Department of Histopathology, Charing Cross Hospital, London and \\ ${ }^{4}$ Department of Histopathology, Royal Marsden Hospital, London, UK
}

\begin{abstract}
Inflammatory myofibroblastic tumours (IMFT) may arise at any anatomical site, including lung, soft tissues, retroperitoneum and bladder. Although morphologically similar, these lesions encompass a spectrum of entities with differing aetiology, ranging from reactive/regenerative proliferations to low-grade neoplasms with a risk of local recurrence, but no significant metastatic potential. Vesical IMFT usually presents as a polypoid mass with a pale firm cut surface and can be of considerable size, mimicking a malignant tumour clinically and radiologically. Its good outcome, however, warrants conservative surgical excision, emphasising the importance of identification and distinction from malignant tumours of the bladder that may require more radical surgery and/or adjuvant therapy. We conducted a preliminary retrospective, comparative immunocytochemical study of 20 bladder tumours, including nine IMFTs, five spindle cell (sarcomatoid) carcinomas, two rhabdomyosarcomas, two leiomyosarcomas and two neurofibromas. The results confirmed IMFT positivity for smooth muscle actin, desmin and cytokeratin in $78-89 \%$ cases, resulting in potential confusion with sarcomatoid carcinoma or leiomyosarcoma. In contrast, cytoplasmic anaplastic lymphoma kinase (ALK 1) staining was present in eight IMFT (89\%), but was not seen in any other lesion examined. The ALK 1 staining was confirmed by fluorescence in situ hybridisation, with translocation of the ALK gene present in $15-60 \%$ tumour cells in four of six IMFT examined, but not in four cases of sarcomatoid carcinoma or three of leiomyosarcoma. In conclusion, ALK 1 staining may be of value in the distinction of vesical IMFT from morphologically similar entities, and often reflects ALK gene translocations in these lesions.
\end{abstract}

Modern Pathology (2004) 17, 765-771, advance online publication, 23 April 2004; doi:10.1038/modpathol.3800078

Keywords: Inflammatory myofibroblastic tumour; ALK 1; bladder

Attributed initially to the lung, ${ }^{1}$ inflammatory myofibroblastic tumours (IMFT) are now known to arise in a wide variety of body sites, including the abdominal cavity, retroperitoneum, pelvis and bladder. ${ }^{2-5}$ In the early literature, these lesions were classified into separate groups based on their anatomical site and postulated aetiology, using terms such as 'inflammatory pseudotumour', 'in-

Correspondence: A Freeman, Department of Histopathology, University College Hospital, University Street, London WC1E 6JJ, UK.

E-mail: afreeman@doctors.org.uk

Received 29 September 2003; revised 9 November 2003; accepted 30 November 2003; published online 23 April 2004 flammatory fibromyxoid tumour', 'postoperative spindle cell nodule' and 'plasma cell granuloma'. ${ }^{6-8}$ More recently, IMFT has been widely accepted as the terminology for a subset of these lesions that are thought to be neoplastic in nature, while other cases thought to be infective, inflammatory or reactive in origin are called 'pseudotumours' ${ }^{9}$

Studies of IMFT at a variety of anatomical sites have shown that these tumours present most frequently in children and young adults (mean age $9-10$ years), with rare cases in middle age. ${ }^{10,11}$ They arise slightly more commonly in females $(\mathrm{M}: \mathrm{F}=3: 4)$ and may reach up to $17 \mathrm{~cm}$ in size (mean $6 \mathrm{~cm}$ ). Clinical presentation is usually as a mass lesion, with symptoms of pain, fever and 
weight loss and haematological findings of anaemia, thrombocytosis, increased erythrocyte sedimentation rate (ESR) and raised gamma globulins. ${ }^{12}$

Three main microscopic patterns are recognised in IMFT: (1) loose stellate cells with a myxoid background including scattered inflammatory cells (nodular fasciitis-like), (2) spindle cells with a compact fascicular pattern, and (3) sparsely cellular collagenised area, and these may be present within the same case. ${ }^{11}$

As a result of endoscopic and microscopic features, including size, presence of atypical spindle cells, scattered mitoses and an 'infiltrative' margin, these entities may be mistaken for malignant spindle cell lesions and the differentials considered vary with the site of the lesion.

In the bladder, the main differential diagnoses for IMFT are spindle cell carcinoma, leiomyosarcoma, neurofibroma ${ }^{13}$ and, particularly in adolescents or young children, rhabdomyosarcoma. Although, these tumours are classically said to be distinguishable on morphological grounds (Table 1), the features enabling distinction may be focal and thus not apparent throughout the tumour, or may be partially obscured by a range of inflammatory and reactive infiltrates or by artefacts of processing or fixation.
Immunohistochemistry may be helpful in the differential diagnosis of spindle cell lesions of the bladder, with each entity showing a different immunophenotypic profile (Table 1). However, there is a wide spectrum of possible immunophenotypes for each entity and an individual case may not show the expected 'classical' immunoprofile. For instance, immunocytochemical studies in recent years have shown that IMFT are strongly positive (usually $>90 \%$ cases) for vimentin and smooth muscle actin (SMA), and variably positive for desmin $(0-69 \%)$, cytokeratin $(20-40 \%)$, macrophage marker (KP 1) and CD30, raising the possibility of confusion with spindle cell carcinoma or sarcoma. ${ }^{2,10,14,15}$

More recently, the presence of anaplastic lymphoma kinase (ALK 1) staining in some cases has been postulated to be of value in the confirmation of IMFT and in its distinction from other entities. A series of studies examining the expression of ALK 1 protein expression by immunocytochemistry or ALK gene rearrangements by fluorescence in situ hybridisation (FISH) in IMFT is shown in Table 2., ${ }^{5,11,16-19}$

The presence of ALK gene translocations involving the 2 p23 band, and the subsequent identification of ALK fusion proteins with the protein

Table 1 Spindle cell vesical lesions-morphological features and 'classical' immunoprofile

\begin{tabular}{|c|c|c|c|c|c|c|c|c|c|}
\hline Entity & Morphological feature & $C K$ & $S M A$ & Des. & Calp & Cald & MyoD1 & $S 100$ & $A L K$ \\
\hline $\begin{array}{l}\text { Spindle cell } \\
\text { carcinoma }\end{array}$ & $\begin{array}{l}\text { Atypia of surface urothelium, } \\
\text { cohesive 'packets' of cells, } \\
\text { marked pleomorphism, } \\
\text { infiltrative margin }\end{array}$ & + & $\begin{array}{l}-/+ \\
(\text { focal, } \\
<25 \% \\
\text { cases) }\end{array}$ & $\begin{array}{l}-/+ \\
(\text { focal, } \\
<25 \% \\
\text { cases) }\end{array}$ & - & - & - & - & - \\
\hline Leiomyo-sarcoma & $\begin{array}{l}\text { Parallel bundles of cells, blunt } \\
\text { ended nuclei, paranuclear } \\
\text { cytoplasmic vacuoles }\end{array}$ & $\begin{array}{l}-/+ \\
\text { (up to } \\
30 \% \text { ) }\end{array}$ & + & + & + & + & - & - & - \\
\hline Neurofibroma & $\begin{array}{l}\text { May be polypoid or diffuse } \\
\text { (plexiform), wavy nuclei }\end{array}$ & - & - & - & - & - & - & + & - \\
\hline Rhabdomyo-sarcoma & $\begin{array}{l}\text { Commonly embryonal type, } \\
\text { monomorphous small round } \\
\text { blue cells }\end{array}$ & $-1+$ & $-/+$ & + & - & - & + & - & $\begin{array}{l}-/+ \\
(20 \%)\end{array}$ \\
\hline IMFT & $\begin{array}{l}\text { Mild nuclear pleomorphism, } \\
\text { myxoid stroma, inflammatory } \\
\text { cells, pushing margin }\end{array}$ & $\begin{array}{c}-/+ \\
(20-40 \%)\end{array}$ & $\begin{array}{c}+/- \\
(\text { over } 90 \%)\end{array}$ & $\begin{array}{c}+/- \\
(0-69 \%)\end{array}$ & $+1-$ & $+/-$ & - & - & $\begin{array}{c}+ \\
(33- \\
75 \%)\end{array}$ \\
\hline
\end{tabular}

$\mathrm{CK}=$ cytokeratin, $\mathrm{SMA}=$ smooth muscle actin, Des. $=$ desmin, Calpo. $=$ calponin, Cald.$=$ caldesmon.

Table 2 Expression of ALK 1 protein and ALK gene rearrangements in IMFT

\begin{tabular}{|c|c|c|c|c|}
\hline Author & Cases & Site & ALK 1 protein expression & $A L K$ gene rearrangement \\
\hline Cook (2001) & 73 IMFT (4 bladder) & Various & $60 \%$ cases $(75 \%$ bladder $)$ & - \\
\hline Chan (2001) & 61 IMFT ( 6 bladder) & Various & $8 \%$ cases ( $33 \%$ bladder) & - \\
\hline Coffin (2001) & 47 IMFT & Various & $36 \%$ cases & $47 \%$ cases \\
\hline Kutok (2001) & 13 IMFT & Spleen, lymph nodes & $0 \%$ cases & - \\
\hline Kapusta (2003) & 12 IMFT & Kidney & $0 \%$ cases & - \\
\hline Hirsch (2003) & 23 IMFT & Genitourinary tract & $38 \%$ cases & $0 \%$ cases \\
\hline
\end{tabular}

$-=$ Not studied 
products of the tropomyosin (TPM3, 4) and clathrin heavy chain genes (CLTC) in a proportion of IMFT, is further evidence that a subset of these entities is indeed neoplastic. ${ }^{20-22}$

The favourable outcome of IMFT in the lung and bladder $^{23}$ is well known, however, and even in a wide range of anatomical sites examined, there is a low rate of local recurrence $(25 \%)$ and no metastases reported (Table 3). ${ }^{2,10,15}$ This indicates that vesical IMFT may be treated with conservative surgical excision compared to that performed with sarcomatous lesions, which may resemble IMFT clinically and radiologically.

We performed a preliminary retrospective immunocytochemical study on nine vesical IMFT and 11 other spindle cell lesions of the bladder, with the aim of assessing ALK1 staining and its value in the distinction of IMFT from other vesical lesions with a similar morphology. Some of the cases were further examined for ALK gene translocations using FISH, and the results were compared with the immunocytochemical expression of the ALK protein.

\section{Materials and methods}

\section{Case Selection}

Nine cases of vesical IMFT were retrieved from the archives of the histopathology departments involved in the project. A number of other vesical spindle cell lesions, including five spindle cell carcinomas (carcinosarcomas), two leiomyosarcomas, two rhabdomyosarcomas and two neurofibromas were also selected for comparison. The ages and clinical features of the nine vesical IMFT cases are presented in Table 4.
The slides were reviewed by AF and MCP, and one representative block was chosen per case for immunocytochemical examination, including normal urothelium and muscularis propria as internal controls where possible. Serial sections $(5 \mu \mathrm{m})$ were cut and stained for a pancytokeratin (CAM 5.2 or MNF116), SMA, desmin, ALK 1, calponin and caldesmon and myogenin for the rhabdomyosarcomas, as shown in Table 5.

\section{Immunocytochemical Method}

The slides were dewaxed in xylene, rehydrated through graded alcohols and rinsed in water. Antigen retrieval was performed using microwaving or enzyme digestion techniques as shown in Table 4 . Endogenous peroxidase activity was blocked for 10 min with DAKO peroxidase block (Catalogue Number S2023). Primary antibodies were then applied for $1 \mathrm{~h}$ at room temperature. The stain was amplified using the DAKO ChemMate Peroxidase/ DAB kit (Catalogue Number K5001) according to the manufacturer's guidelines. The slides were finally washed in water, taken through graded alcohols and xylene and coverslips applied.

\section{Interpretation of Staining}

The staining pattern of the slides was examined using a semiquantitative scoring method by AF and MCP. The slide was scored as 'positive' or 'negative' according to the pattern of staining seen and the extent quantitated as 'focal' or 'diffuse'. The term 'focal' was used to indicate a patchy staining pattern, with $<25 \%$ of all cells staining with the

Table 3 Studies of outcome in IMFT at various sites, including the bladder

\begin{tabular}{llllcrr}
\hline Author & Year & Cases & Site & Mean follow up (months) & Local recurrence (\%) & Metastases (\%) \\
\hline Jones & 1993 & 13 IMFT & Bladder & 25 & 0 & 0 \\
Coffin & 1995 & 84 IMFT & Various & 24 & 25 & 0 \\
Iczkowski & 2001 & 17 IMFT & Bladder & 35 & 0 \\
\hline
\end{tabular}

Table 4 Clinicopathological information for cases of vesical IMFT

\begin{tabular}{|c|c|c|c|c|c|}
\hline Case & Age (Years) & Sex & Size & Treatment & Follow-up \\
\hline 1 & 50 & $\mathrm{M}$ & Not known & Cystectomy & - \\
\hline 2 & 36 & $\mathrm{~F}$ & $7.5 \mathrm{~cm}$ polyp & Cystectomy & - \\
\hline 3 & 51 & $\mathrm{~F}$ & Not known & Cystectomy & - \\
\hline 4 & 13 & $\mathrm{M}$ & $5.3 \mathrm{~cm}$ polyp & Partial cystectomy & 4 years FOD \\
\hline 5 & 34 & $\mathrm{~F}$ & $4.1 \mathrm{~cm}$ polyp & Partial cystectomy & 2 years FOD \\
\hline 6 & 46 & $\mathrm{M}$ & $5.0 \mathrm{~cm}$ polyp & Partial cystectomy & 2 vears FOD \\
\hline 7 & 33 & $\mathrm{M}$ & $2.0 \mathrm{~cm}$ & Partial cystectomy & 8 months FOD \\
\hline 8 & 36 & $\mathrm{~F}$ & $4.0 \mathrm{~cm}$ & Partial cystectomy & 8 months FOD \\
\hline 9 & 30 & M & $>5.0 \mathrm{~cm}$ & TUR & 5 months FOD \\
\hline
\end{tabular}

$-=$ follow-up not available, FOD $=$ free of disease, $\mathrm{TUR}=$ transurethral resection. 
Table 5 Antibodies used in the current study

\begin{tabular}{lllll}
\hline Antibody & Manufacturer & Antigen retrieval & Antibody dilution & Incubation time $(h)$ \\
\hline MNF 116 & DAKO & 10 min CT & $1 / 100$ & 1 \\
CAM 5.2 & Becton Dickinson & 10 min CT & $1 / 2$ & 1 \\
SMA & DAKO & No treatment & $1 / 150$ & 1 \\
Desmin & DAKO & PH 6.0 MW & $1 / 50$ & 1 \\
ALK 1 & DAKO & High pH MW & $1 / 3000$ & 1 \\
Calponin & A Menarini & PC & $1 / 100$ & 1 \\
Caldesmon & A Menarini & PC & $1 / 200$ & 1 \\
Myogenin & DAKO & PC & & 1
\end{tabular}

$10 \mathrm{CT}=10$ min with $0.1 \%$ chymotrypsin (Sigma C4129) at $37^{\circ} \mathrm{C}$, pH 7.8 ; High $\mathrm{pH} \mathrm{MW}=25$ min high power in an $800 \mathrm{~W}$ microwave in $\mathrm{DAKO}$ target retrieval solution High pH (DAKO S3307), followed by 10 min standing time; pH $6.0 \mathrm{MW}=25 \mathrm{~min}$ high power in an $800 \mathrm{~W}$ microwave in DAKO target retrieval solution (DAKO S1699), followed by $10 \mathrm{~min}$ standing time; and PC = 2 min at full pressure in a 15 lb pressure cooker in $3 \mathrm{l}$ of $0.01 \mathrm{M}$ citrate buffer, $\mathrm{pH}$ 6.0.

applied marker, while the term 'diffuse' was used to indicate strong staining in over $25 \%$ of cells. The entire slide was examined in an attempt to account for tumour heterogeneity. Positive controls were examined with each batch of stained slides for standardisation of scoring, in addition to the urothelium and muscularis internal tissue controls, and negative controls were performed by omitting the primary antibody.

\section{FISH Protocol}

Paraffin-embedded tissue sections were processed using the Vysis Paraffin Pretreatment Kit protocol, substituting xylene in place of the Hemo-De clearing agent. Protease times used were between 30 and 100 min. Slides were denatured in $70 \%$ formamide/ $2 \times \mathrm{SSC}$ at $75^{\circ} \mathrm{C}$ for $6 \mathrm{~min}$ followed by dehydration in an ethanol series. FISH was performed with the Vysis LSI ALK probe according to the manufacturer's instructions (Vysis). The probe set contains a $250 \mathrm{bp}$ probe telomeric to the breakpoint region, labelled in Spectrum Orange, and a $300 \mathrm{bp}$ probe centromeric to the breakpoint region, labelled in Spectrum Green. Slides were examined using the BX50 Olympus microscope, equipped with appropriate filters. At least 150 interphases were examined per slide and the percentage of cells showing separation of the probes, reflecting ALK gene translocation, was calculated.

When hybridised with the LSI ALK Dual Color, Break Apart Rearrangement Probe, the 2p23 ALK region in its native state will be seen as two immediately adjacent or fused orange/green (yellow) signals (Figure 2b). However, if a $t(2 ; 5)$ or other chromosome rearrangement at the 2p23 ALK breakpoint region has occurred, one orange and one green signal will be seen, while the native ALK region will remain as an orange/green fusion signal (Figure 2a). The hybridisation result of the LSI ALK Dual Color, Break Apart Rearrangement Probe containing the $t(2 ; 5)$ will be the centromeric green probe remaining at $2 p 23$, while the telomeric orange signal that covers the region is translocated to $5 \mathrm{q} 35$ on the derivative chromosome.

\section{Results}

\section{Immunocytochemistry (Table 6, Figure 1)}

A positive cytokeratin immunostain was seen in 8/9 (89\%) IMFT, all five spindle cell carcinomas (100\%) and $1 / 2(50 \%)$ leiomyosarcomas. SMA was positive in $8 / 9(89 \%)$ IMFT, $4 / 5(80 \%)$ spindle cell carcinomas and 2/2 (100\%) leiomyosarcomas (Figure 1, Table 1). In contrast, desmin was positive in $7 / 9$ (78\%) IMFT, $2 / 5$ (40\%) spindle cell carcinomas and $2 / 2(100 \%)$ rhabdomyosarcomas, but negative in leiomyosarcoma.

Calponin and caldesmon, novel markers for smooth muscle tumours, were positive in $2 / 2$ $(100 \%)$ leiomyosarcomas, and but also in $8 / 9$ $(89 \%)$ and 6/9 (67\%) IMFT, respectively, indicating their presence in myofibroblasts as well as smooth muscle fibres.

ALK 1 staining revealed a cytoplasmic pattern of staining in 8/9 IMFT (89\%), but was not seen in any of the spindle cell carcinomas, leiomyosarcomas or rhabdomyosarcomas examined.

\section{FISH Results}

Six IMFT, four sarcomatoid carcinomas and three leiomyosarcomas were examined for ALK gene rearrangements, using the commercial probe kit from Vysis as explained above.

Four of six IMFT (67\%) showed ALK gene rearrangements, as characterised by separation of the centromeric and telomeric probes on either side of the common ALK breakpoint region onto different chromosomes, in a range of between 15 and $60 \%$ of the tumour cells examined. In contrast, the sarcomatoid carcinomas and leiomyosarcomas examined were completely negative for ALK translocations (Figure 2).

One of the IMFT cases examined revealed an inversion of Chromosome 2 (inv. 2 p23q35) resulting 
Table 6 Results of immunocytochemical staining

\begin{tabular}{|c|c|c|c|c|c|c|}
\hline Type of tumour & Case & $C K(M N F 116$ or $C A M)$ & $S M A$ & Desmin & Calponin, Caldesmon & $A L K 1$ \\
\hline IMFT & 9 & $8(5 \mathrm{~F}, 3 \mathrm{D})$ & $8(5 \mathrm{~F}, 3 \mathrm{D})$ & 7 (all F) & 8 (5 F, 3D) 6 (4 F, 2D) & $8(3 \mathrm{~F}, 4 \mathrm{D})$ \\
\hline Sarcomatoid carcinoma & 5 & $5($ all F) & $4(2 \mathrm{~F}, 2 \mathrm{D})$ & $2(1 \mathrm{~F}, 1 \mathrm{D})$ & - & 0 \\
\hline Rhabdomyosarcoma $^{\mathrm{a}}$ & 2 & 0 & 0 & 2 & - & 0 \\
\hline Leiomyosarcoma & 2 & $1(1 \mathrm{~F})$ & $2(1 \mathrm{~F}, 1 \mathrm{D})$ & 0 & 2 (all F) $2(1 \mathrm{~F}, 1 \mathrm{D})$ & 0 \\
\hline Neurofibroma & 2 & 0 & 0 & - & - & 0 \\
\hline
\end{tabular}

$\mathrm{F}=$ focal, $\mathrm{D}=$ diffuse, $-=$ not done.

anuclear positivity for myogenin.
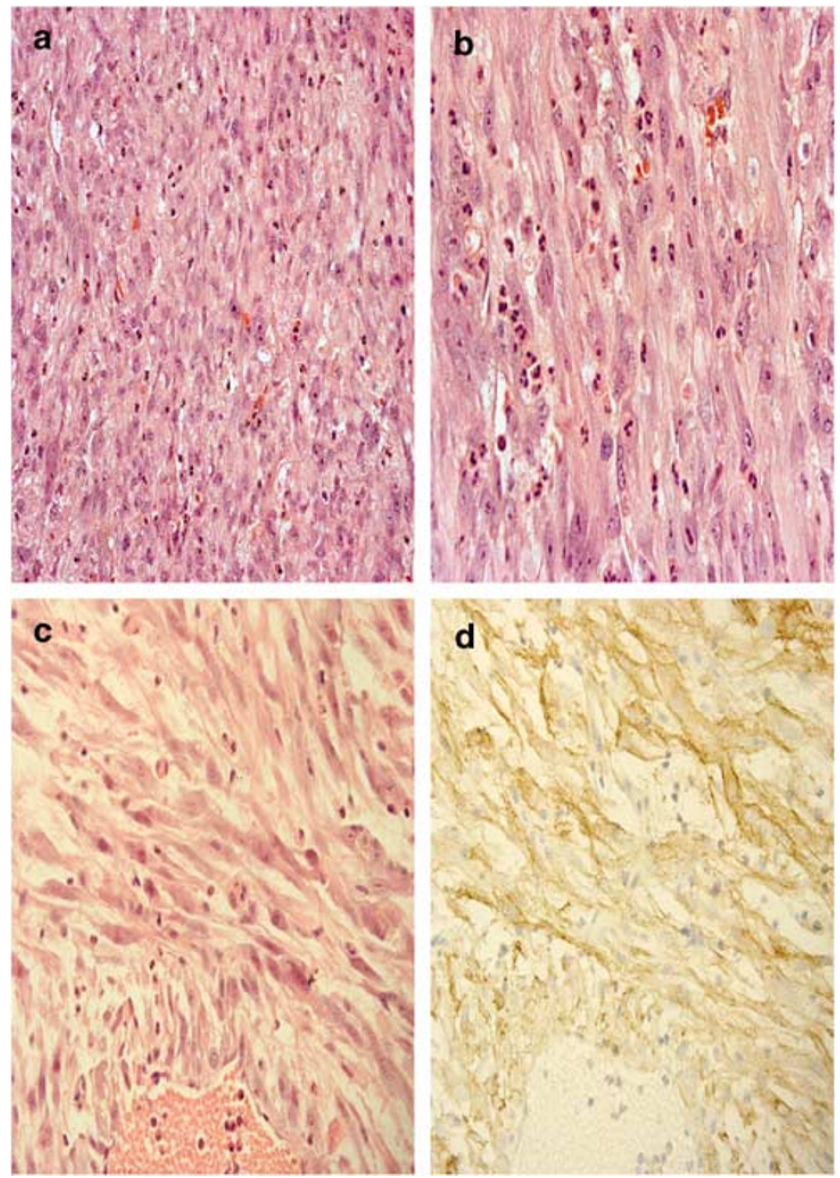

Figure 1 An IMFT showing compact fascicles of spindle cells, with an inflammatory component of (a) $\times 200$ magnification (b) and $\times 400$ magnification. (c) Areas of looser spindled cells in a myxoid background are focally seen $(\times 400$ magnification). (d) The ALK 1 immunostain shows diffuse cytoplasmic positivity ( $\times 400$ magnification) in the neoplastic cells.

in a variant fusion protein between the ALK gene and ATIC gene. This translocation has previously been reported in anaplastic large cell lymphoma but not, as far as we are aware, in IMFT.

\section{Discussion}

IMFT are a group of lesions that arise in a variety of anatomical sites, and are postulated to be aetiologically composed of a variety of entities that may be either neoplastic or inflammatory in nature. The
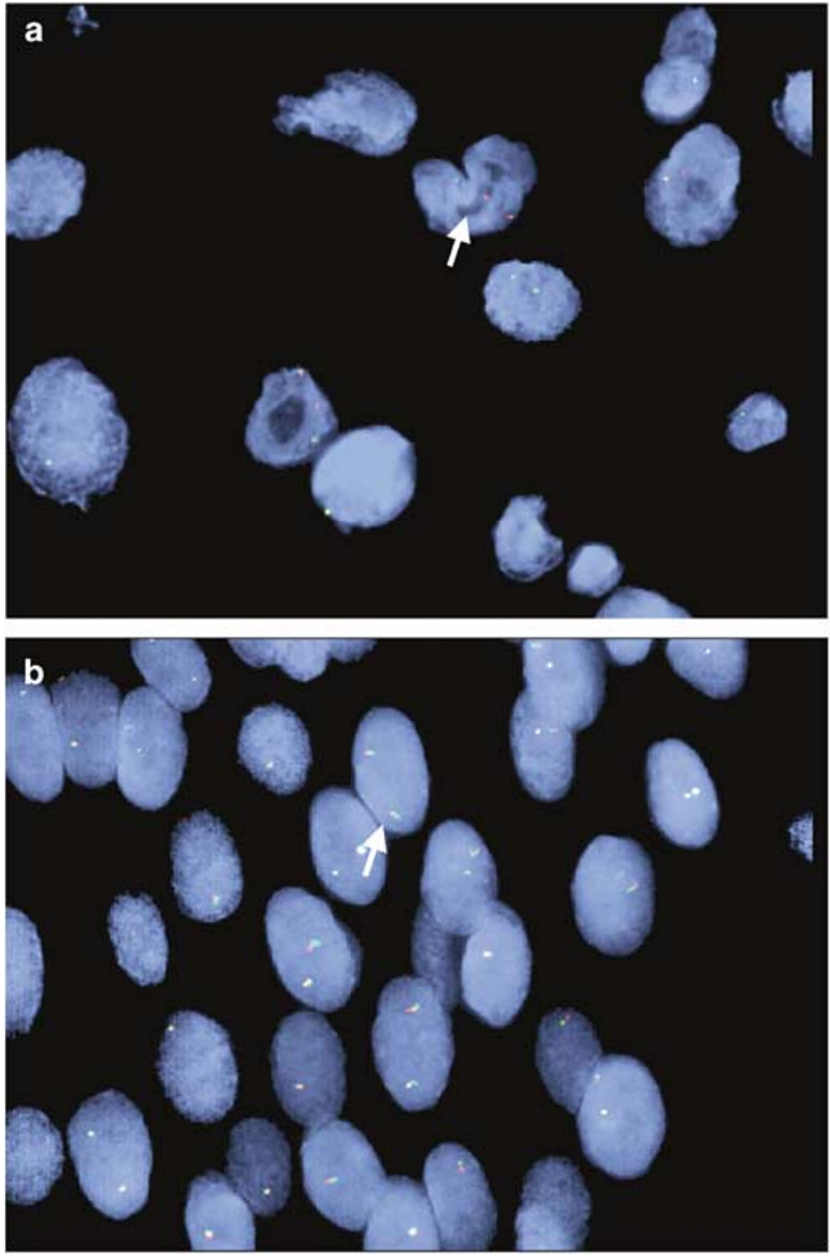

Figure 2 (a) FISH studies confirm the presence of ALK gene rearrangement in the cells of an IMFT. There is displacement of the green and orange probes against the centromeric and telomeric portion of the common breakpoint region of the ALK gene onto different chromosomes at one allele (white arrow). This shows that part of the ALK gene is translocated from Chromosome $2 p$ onto Chromosome 5. (b) Cells of a leiomyosarcoma show an intact ALK gene at both alleles, with the green and orange probes adjacent to each other (white arrow). This finding confirms that the centromeric and telomeric portion of the ALK gene are on the same chromosome, excluding the presence of a gene translocation at this point.

favourable outcome of most of these lesions, particularly in the lung and bladder is well known, and has implications for initial surgical resection and follow-up. However, the clinical and 
radiological similarity of vesical IMFT to sarcomas and spindle cell carcinomas may cause a potential diagnostic problem.

At present, the morphological criteria suggestive of IMFT include a variably cellular spindle cell tumour composed of reactive myofibroblastic cells with elongated nuclei, and the presence of stellate cells, with scattered mitoses but no significant atypia or necrosis. These features are not totally specific to IMFT and may be focally seen in some sarcomas. Electron microscopy is also not definitive, although quantitative differences in cytoskeletal filaments between smooth muscle cells and myofibroblasts may be useful in some cases, and the presence of a fibronexus junction, although rarely visualised by electron microscopy, is also indicative of a myofibroblastic phenotype. ${ }^{24,25}$

Immunocytochemistry using conventional markers for smooth muscle and epithelial markers has been shown to be positive in a proportion of IMFT, making the evaluation of positive staining for SMA, desmin and cytokeratin unreliable in their distinction from leiomyosarcoma and carcinoma with spindle cell change.

In recent years, ALK 1 has been found to be expressed in the cytoplasm of IMFT in a variable proportion of cases. Studies of ALK 1 protein using immunohistochemical methods have shown positive staining in a range of $0-75 \%$ of cases, with high rates of expression in vesical IMFT (33-75\%), and complete absence in IMFT of the spleen and lymph nodes. . $^{511,16-18}$

The results of our preliminary study using nine vesical IMFT support these findings, with ALK 1 protein immunocytochemically identifiable in eight vesical IMFT (89\%), but negative in all 11 sarcomatoid carcinoma, leiomyosarcoma, rhabdomyosarcoma and neurofibromas examined from the bladder. Our results agree with a recent abstract that showed cytoplasmic ALK 1 staining in 12/16 (75\%) IMFT, but not in 15 control cases, including leiomyosarcoma, stromal tumours and carcinosarcomas. ${ }^{26}$

Although a number of cases of rhabdomyosarcoma may show expression of ALK 1, particularly those of alveolar subtype, ${ }^{27}$ these lesions usually also express desmin and myogenin or myoD1, and show characteristic morphological features that allow identification and distinction from IMFT.

The immunocytochemical ALK 1 protein expression is reflected in the results of the FISH studies. ALK gene translocations were present in a percentage of tumour cells in 4/6 IMFT examined (67\%), indicating that a genetic abnormality in the area of Chr. 2p23 often results in overexpression of the ALK 1 protein, and may be an important factor in tumorigenesis of IMFT. In contrast, none of the seven other bladder spindle cell lesions examined showed translocation in the ALK gene, confirming the negative result by immunocytochemistry. The absence of an identifiable ALK translocation in the remaining two IMFT tested may either reflect the sensitivity of the technique, or be attributed to the fact that the commercial probe used in this experiment only identifies translocations at the most common site of the ALK gene and may not show translocations at other sites. Our finding correlates well with the results of another group, who found $47 \%$ ALK gene rearrangement in IMFT, ${ }^{16}$ but is in contrast with those of a recent abstract that shows $0 \%$ ALK gene rearrangement in 23 genitourinary cases studied. ${ }^{19}$ Although the number of cases examined in each category of our study was small, there is no obvious confounding factor in the series of cases examined, and thus no reason why these results may not be extrapolated to a diagnostic setting.

In summary, ALK 1 protein is often upregulated in vesical IMFT by translocation of the ALK gene and is identifiable by immunocytochemistry, making it a valuable marker in cases of IMFT in the bladder. The expression of ALK is not seen in the majority of cases of sarcomatoid carcinomas, leiomyosarcomas or rhabdomyosarcomas of the bladder. Positive ALK 1 immunocytochemical staining, with the conventional morphological and ultrastructural features in current use, may be of value in the distinction of IMFT from other spindle cell lesions of the bladder. The presence of ALK gene translocation may provide further evidence for a neoplastic rather than a reactive or inflammatory nature for the lesion and may be useful in indicating which lesions may show local recurrence potential.

\section{References}

1 Umiker WO, Iverson L. Postinflammatory 'tumour' of the lung. Report of four cases simulating xanthoma, fibroma or plasma cell granuloma. J Thorac Cardiovasc Surg 1954;28:55-63.

2 Jones EC, Clement PB, Young RH. Inflammatory pseudotumour of the bladder: a clinicopathological, immunohistochemical, ultrastructural and flow cytometric study of 13 cases. Am J Surg Pathol 1993; 17:264-274.

3 Coyne JD, Wilson G, Sandhu D, et al. Inflammatory pseudotumour of the urinary bladder. Histopathology 1991;18:261-264.

4 Coffin CM, Humphrey PA, Dehner LP. Extrapulmonary myofibroblastic tumour: a clinical and pathologic survey. Semin Diagn Pathol 1998;15:85-101.

5 Chan JKC, Cheuk W, Shimizu M. Anaplastic lymphoma kinase expression in inflammatory pseudotumour. Am J Surg Pathol 2001;25:761-768.

6 Jufe R, Molinolo AA, Fefer SA, et al. Plasma cell granuloma of the bladder: a case report. J Urol 1984; 131:1175-1176.

7 Nochomovitz LE, Orenstein JH. Inflammatory pseudotumour of the urinary bladder - possible relationship to nodular fasciitis. Two case reports, cytologic observations and ultrastructural observations. Am J Surg Pathol 1985;9:366-373. 
8 Chan JKC. Inflammatory pseudotumour: a family of lesions of diverse nature and aetiologies. Adv Anat Pathol 1996;3:156-171.

9 Arber DA, Kamel OW, van de Rijn M, et al. Frequent presence of the Epstein-Barr virus in inflammatory pseudotumour. 1995;26:1093-1098.

10 Coffin CM, Watterson J, Priest JR, et al. Extrapulmonary inflammatory myofibroblastic tumour (inflammatory pseudotumour): a clinicopathologic and immunohistochemical study of 84 cases. Am J Surg Pathol 1995;19:859-872.

11 Cook JR, Dehner LP, Collins MH, et al. Anaplastic lymphoma kinase (ALK) expression in the inflammatory myofibroblastic tumour. Am J Surg Pathol 2001; 25:1364-1371.

12 Petinato G, Manivel JC, de Rosa N, et al. Inflammatory myofibroblastic tumour (plasma cell granuloma): clinicopathologic study of 20 cases with immunohistochemical and ultrastructural observations. Am J Clin Pathol 1990;94:538-546.

13 Cheng L, Scheithauer BW, Leibovich BC, et al. Neurofibroma of the urinary bladder. Cancer 1999;86:505-513.

14 Lundgren L, Aldenborg F, Angervall L, et al. Pseudomalignant spindle cell proliferations of the urinary bladder. Human Pathol 1994;25:181-191.

15 Iczkowski KA, Shanks JH, Gadaleanu V, et al. Inflammatory pseudotumour and sarcoma of urinary bladder: differential diagnosis and outcome in thirtyeight spindle cell neoplasms. Mod Pathol 2001;14: 1043-1051.

16 Coffin CM, Patel A, Perkins S, et al. ALK and p80 expression and chromosomal rearrangements involving 2p23 in inflammatory myofibroblastic tumour. Mod Pathol 2001;14:569-576.

17 Kutok JL, Pinkus GS, Dorfman DM, et al. Inflammatory pseudotumour of lymph nodes and spleen: an entity biologically distinct from inflammatory myofibroblastic tumour. Human Pathol 2001;32:1382-1387.

18 Kapusta LR, Weiss MA, Ramsay J, et al. Inflammatory myofibroblastic tumours of the kidney: a clinicopathologic and immunohistochemical study of 12 cases. Am J Surg Pathol 2003;27:658-666.

19 Hirsch MS, Cin PD, Fletcher CDM. ALK expression in reactive pseudosarcomatous myofibroblastic proliferations of the genitourinary tract, USCAP Meeting Abstr 697: 2003;153A.

20 Lawrence B, Perez-Atayde A, Hibbard MK, et al. TPM3-ALK and TPM4-ALK oncogenes in inflammatory myofibroblastic tumours. Am J Pathol 2000;157: $377-384$

21 Bridge JA, Kanamori M, Ma Z, et al. Fusion of the ALK gene to the clathrin heavy chain gene CLTC in inflammatory myofibroblastic tumour. Am J Pathol 2001;159:411-415.

22 Griffin CA, Hawkins AL, Dvorak C, et al. Recurrent involvement of 2p23 in inflammatory myofibroblastic tumours. Cancer res 1999;59:2776-2780.

23 Colby TV, Koss MN, Travis WD. Tumours of the lower respiratory tract, In: Colby, Koss, Travis (eds). Atlas of Tumour Pathol Armed Forces Institute of Pathology: Washington, 1995, pp 327-341.

24 Eyden B. The fibronexus in reactive and tumoral myofibroblasts: further characterisation by electron microscopy. Histol Histopathol 2001;16:57-70.

25 Eyden B. Electron microscopy in the study of myofibroblastic lesions. Semin Diagn Pathol 2003;20:13-24.

26 Tsuzuki T, Magi-Galluzzi C, Epstein JI. ALK 1 expression in pseudosarcomatous myofibroblastic tumor (PSFMT) of the urinary bladder, USCAP Meeting Abstr 795: 2003;p174A.

27 Pillay K, Govender G, Chetty R. ALK protein expression in rhabdomyosarcomas. Histopathology 2002;41: $461-467$ 